\title{
Existence Results for a Class of Periodic Evolution Variational Inequalities
}

\author{
Samir ADLY, Daniel GOELEVEN, Michel THERA
}

\begin{abstract}
In this paper, using the Brouwer topological degree, the authors prove an existence result for finite variational inequalities. This approach is also used to obtain the existence of periodic solutions for a class of evolution variational inequalities.
\end{abstract}

Keywords Variational inequalities, Differential inclusions, Topological degree, Guiding functions, Periodic solutions

2000 MR Subject Classification 49J40, 49J20, 35K85

\section{Introduction}

It has been well recognized that variational inequalities offer the right framework to consider numerous applied problems in various areas such as economics and engineering. Throughout this paper, we consider $\mathbb{R}^{n}$ equipped with the usual euclidean scalar product $\langle\cdot, \cdot\rangle$. We start by considering a variational inequality $\operatorname{VI}(\Lambda, \varphi)$, that is, the problem of finding $\bar{x} \in \mathbb{R}^{n}$ such that

$$
\langle\Lambda(\bar{x}), v-\bar{x}\rangle+\varphi(v)-\varphi(\bar{x}) \geq 0, \quad \forall v \in \mathbb{R}^{n} .
$$

In this formulation, $\Lambda: \mathbb{R}^{n} \rightarrow \mathbb{R}^{n}$ is a continuous map, $\varphi: \mathbb{R}^{n} \rightarrow \mathbb{R}$ is a convex function. When the operator $\Lambda$ under consideration is coercive, existence results for the problem $\operatorname{VI}(\Lambda, \varphi)$ are well known in the setting of reflexive Banach spaces. This study was initiated by G. Stampacchia in the 60's, and we refer to the contributions of J. L. Lions [15], Brézis [4] and Kinderlehrer and Stampacchia [12] for various results and references therein.

In the first part of this paper, we develop an original approach essentially based on the use of the Brouwer topological degree to prove results related to the existence of a solution to problem $\operatorname{VI}(\Lambda, \varphi)$.

Then, we study a first order evolution variational inequality, that is, a differential inclusion of the form: find a $T$-periodic function $u \in C^{0}\left([0, T] ; \mathbb{R}^{n}\right)$ such that

$$
\frac{d u}{d t}(t)+F(u(t))-f(t) \in-\partial \varphi(u(t)), \quad \text { a.e. } t \in[0, T],
$$


where $F: \mathbb{R}^{n} \rightarrow \mathbb{R}^{n}$ is a continuous map, $\varphi: \mathbb{R}^{n} \rightarrow \mathbb{R}$ is a convex function, $f \in C^{0}\left(\left[0,+\infty\left[; \mathbb{R}^{n}\right)\right.\right.$ is such that $\frac{d f}{d t} \in L_{\text {loc }}^{1}\left(0,+\infty ; \mathbb{R}^{n}\right), T>0$ is a prescribed period and $\partial \varphi$ is the convex subdifferential operator. This problem is studied by means of a continuation method. It is well known that the Brouwer topological degree plays a fundamental role in the theory of ordinary differential equations (ODE). M. A. Krasnosel'skii [13, 14] and H. Amann [3], developed a continuation method to compute this Brouwer topological degree associated to some gradient mapping (called the method of guiding function). This approach was useful for the study of the existence of periodic solutions for ODE's. Roughly speaking, if on some balls of $\mathbb{R}^{n}$ the Brouwer topological degree of the Poincaré translation operator (see e.g. [17]) associated to the ODE is different from zero, then the problem has at least one periodic solution (for more details, references and possible extensions to the Leray-Schauder degree, we refer to the monograph of J. Mawhin [17]). With the emergence of many engineering disciplines and due to the lack of smoothness in many applications, it is not surprising that these classical mathematical tools require natural extensions (for both analytical and numerical methods) to the class of unilateral dynamical systems. It is well known that the mathematical formulation of unilateral dynamical systems involves inequality constraints and hence contains natural non-smoothness. In mechanical systems, this non-smoothness could have its origin in the environment of the system studied (e.g. case of contact) in the dry friction, or in the discontinuous control term. Recently, new analytical tools have been developed for the study of unilateral evolution problems (see e.g. $[1,2,7-9]$ and the references therein).

The study of periodic solutions of evolution variational inequalities is also important. The Krasnol'skii's original approach for ODE, has known some extensions in order to obtain continuation methods for differential inclusions (see [10] and the references therein for more details).

In Section 4, we will be concerned with the existence of a $T$-periodic solution $u \in C^{0}([0, T]$; $\left.\mathbb{R}^{n}\right)$ such that

$$
\begin{aligned}
& \frac{d u}{d t} \in L^{\infty}\left(0, T ; \mathbb{R}^{n}\right), \\
& u \text { is right-differentiable on }[0, T), \\
& u(0)=u(T), \\
& \left\langle\frac{d u}{d t}(t)+F(u(t))-f(t), v-u(t)\right\rangle+\varphi(v)-\varphi(u(t)) \geq 0, \quad \forall v \in \mathbb{R}^{n}, \text { a.e., } t \in[0, T] .
\end{aligned}
$$

In this formulation, $F: \mathbb{R}^{n} \rightarrow \mathbb{R}^{n}$ is a continuous map, $\varphi: \mathbb{R}^{n} \rightarrow \mathbb{R}$ is a convex function, $f \in C^{0}\left(\left[0,+\infty\left[; \mathbb{R}^{n}\right)\right.\right.$ is such that $\frac{d f}{d t} \in L_{\text {loc }}^{1}\left(0,+\infty ; \mathbb{R}^{n}\right)$ and $T>0$ is a prescribed period.

We prove (see Corollary 5.1) that if $F$ and $\varphi$ satisfy some growth condition (see (5.17)), then problem (1.1) has at least one periodic solution.

This approach is also used to obtain the existence of a $T$-periodic solution of a second order dynamical system of the form:

$$
M \ddot{q}(t)+C \dot{q}(t)+K q(t)-F(t) \in-H_{1} \partial \Phi\left(H_{1}^{T} \dot{q}(t)\right),
$$

where $q \in \mathbb{R}^{m}$ is the vector of generalized coordinate, $\Phi: \mathbb{R}^{l} \rightarrow \mathbb{R}$ is a convex function, $M \in \mathbb{R}^{m \times m}$ is a symmetric and positive definite matrix, $C \in \mathbb{R}^{m \times m}, K \in \mathbb{R}^{m \times m}$ are given matrices and $H_{1} \in \mathbb{R}^{m \times l}$ is a given matrix whose coefficients are determined by the directions 
of friction forces. The function $F \in C^{0}\left([0,+\infty) ; \mathbb{R}^{m}\right)$ is such that $\frac{d F}{d t} \in L_{\text {loc }}^{1}\left([0,+\infty) ; \mathbb{R}^{m}\right)$. The term $H_{1} \partial \Phi\left(H_{1}^{T} \cdot\right)$ is used to modelise the convex unilateral contact induced by friction forces.

The paper is organized as follows: Section 2 contains some background materials on properties of the Brouwer topological degree and the concept of resolvent operator associated to a subdifferential operator. In Section 3, using an equivalent fixed point formulation as well as the Brouwer topological degree, we give some existence results for finite variational inequalities. In Section 4, we introduce the Poincaré operator associated to problem (1.1). Section 5 is devoted to the existence of a periodic solution of problem (1.1). In Section 6, we show that our approach could be applied to a special second order problem (1.2).

\section{Brouwer Topological Degree and the Resolvent Operator $\boldsymbol{P}_{\lambda, \varphi}$}

It is well known that the degree theory is one of the most powerful tool in nonlinear analysis for the study of zeros of a continuous operator. Let $\Omega \subset \mathbb{R}^{n}$ be an open and bounded subset with boundary $\partial \Omega$ and $f \in C^{1}\left(\Omega ; \mathbb{R}^{n}\right) \cap C^{0}\left(\bar{\Omega}, \mathbb{R}^{n}\right)$. The Jacobian matrix of $f$ at $x \in \Omega$ is defined by $f^{\prime}(x)=\left(\partial_{x_{i}} f_{j}(x)\right)_{1 \leq i, j \leq n}$ and the Jacobian determinant of $f$ at $x \in \Omega$ is defined by

$$
J_{f}(x)=\operatorname{det}\left(f^{\prime}(x)\right)
$$

We set

$$
A_{f}(\Omega)=\left\{x \in \Omega: J_{f}(x)=0\right\}
$$

Observe that if $f^{-1}(0) \cap A_{f}(\Omega)=\emptyset$ and $0 \notin f(\partial \Omega)$, then the set $f^{-1}(0)$ is finite. The quantity $\sum_{x \in f^{-1}(0)} \operatorname{sign}\left(J_{f}(x)\right)$ is therefore defined and is called the Brouwer topological degree of $f$ with respect to $\Omega$ and 0 and is denoted by $\operatorname{deg}(f, \Omega, 0)$. More generally, if $f \in C^{0}\left(\bar{\Omega} ; \mathbb{R}^{n}\right)$ and $0 \notin f(\partial \Omega)$, then the Brouwer topological degree of $f$ with respect to $\Omega$ and 0 , denoted by $\operatorname{deg}(f, \Omega, 0)$, is well defined (see [16] for more details).

In the sequel, we use standard notations. We denote by $\langle\cdot, \cdot\rangle$ the scalar product on $\mathbb{R}^{n}$ and by $\|\cdot\|$ the associated norm. For $r>0$, we set $\mathbb{B}_{r}:=\left\{x \in \mathbb{R}^{n}:\|x\|<r\right\}$, and respectively $\overline{\mathbb{B}}_{r}=\left\{x \in \mathbb{R}^{n}:\|x\| \leq r\right\}$, for the open (respectively closed) unit ball with radius $r>0$. We use the notation $\partial \mathbb{B}_{r}$ to denote the boundary $\overline{\mathbb{B}}_{r} \backslash \stackrel{\circ}{\mathbb{B}_{r}}$ of $\mathbb{B}_{r}$, that is, $\left\{x \in \mathbb{R}^{n}:\|x\|=r\right\}$.

If $f: \overline{\mathbb{B}}_{r} \rightarrow \mathbb{R}^{n}$ is continuous and $0 \notin f\left(\partial \mathbb{B}_{r}\right)$, then the Brouwer topological degree of $f$ with respect to $\mathbb{B}_{r}$ and 0 is well-defined (see e.g. [16]) and denoted by $\operatorname{deg}\left(f, \mathbb{B}_{r}, 0\right)$.

Let us now recall some properties of the topological degree that we will use later.

P1 If $0 \notin f\left(\partial \mathbb{B}_{r}\right)$ and $\operatorname{deg}\left(f, \mathbb{B}_{r}, 0\right) \neq 0$, then there exists $x \in \mathbb{B}_{r}$ such that $f(x)=0$.

P2 Let $\varphi:[0,1] \times \overline{\mathbb{B}}_{r} \rightarrow \mathbb{R}^{n} ;(\lambda, x) \rightarrow \varphi(\lambda, x)$ be continuous such that, for each $\lambda \in[0,1]$, one has $0 \notin \varphi\left(\lambda, \partial \mathbb{B}_{r}\right)$, then the map $\lambda \rightarrow \operatorname{deg}\left(\varphi(\lambda, \cdot), \mathbb{B}_{r}, 0\right)$ is constant on $[0,1]$.

P3 Let us denote by $\operatorname{id}_{\mathbb{R}^{n}}$ the identity mapping on $\mathbb{R}^{n}$. We have

$$
\operatorname{deg}\left(\operatorname{id}_{\mathbb{R}^{n}}, \mathbb{B}_{r}, 0\right)=1
$$


$\mathbf{P 4}$ If $0 \notin f\left(\partial \mathbb{B}_{r}\right)$ and $\alpha>0$, then

$$
\begin{aligned}
\operatorname{deg}\left(\alpha f, \mathbb{B}_{r}, 0\right) & =\operatorname{deg}\left(f, \mathbb{B}_{r}, 0\right), \\
\operatorname{deg}\left(-\alpha f, \mathbb{B}_{r}, 0\right) & =(-1)^{n} \operatorname{deg}\left(f, \mathbb{B}_{r}, 0\right) .
\end{aligned}
$$

P5 If $0 \notin f\left(\partial \mathbb{B}_{r}\right)$ and $f$ is odd on $\mathbb{B}_{r}$ (i.e., $\left.f(-x)=-f(x), \forall x \in \mathbb{B}_{r}\right)$, then $\operatorname{deg}\left(f, \mathbb{B}_{r}, 0\right)$ is odd.

P6 Let $f(x)=A x-b$, with $A \in \mathbb{R}^{n \times n}$ a nonsingular matrix and $b \in \mathbb{R}^{n}$. Then $\operatorname{deg}\left(f, A^{-1} b+\mathbb{B}_{r}, 0\right)=\operatorname{sign}(\operatorname{det} A)= \pm 1$.

Let $V \in C^{1}\left(\mathbb{R}^{n} ; \mathbb{R}\right)$ and suppose that there exists $r_{0}>0$ such that for every $r \geq r_{0}$, $0 \notin \nabla V\left(\partial \mathbb{B}_{r}\right)$. Then $\operatorname{deg}\left(\nabla V, \mathbb{B}_{r}, 0\right)$ is constant for $r \geq r_{0}$ and one defines the index of $V$ at infinity "ind $(V, \infty)$ " by

$$
\operatorname{ind}(V, \infty):=\operatorname{deg}\left(\nabla V, \mathbb{B}_{r}, 0\right), \quad \forall r \geq r_{0}
$$

Let $\varphi: \mathbb{R}^{n} \rightarrow \mathbb{R}$ be a convex mapping. It is well known that

(a) $\varphi$ is continuous on $\mathbb{R}^{n}$;

(b) For all $x \in \mathbb{R}^{n}$, the convex subdifferential of $\varphi$ at $x$ is a nonempty compact and convex subset of $\mathbb{R}^{n}$ and defined by

$$
\partial \varphi(x)=\left\{w \in \mathbb{R}^{n}: \varphi(v)-\varphi(x) \geq\langle w, v-u\rangle, \forall v \in \mathbb{R}^{n}\right\}
$$

(c) For all $x \in \mathbb{R}^{n}$, the directional derivative of $\varphi$ at $x \in \mathbb{R}^{n}$ in the direction $\xi \in \mathbb{R}^{n}$, i.e.,

$$
\varphi^{\prime}(x ; \xi)=\lim _{\alpha \downarrow 0} \frac{\varphi(x+\alpha \xi)-\varphi(x)}{\alpha}
$$

exists (see e.g. [11, p. 164]).

Since the subdifferential operator $\partial \varphi$ associated to $\varphi$, is maximal monotone (see [5]), the operator $(I+\lambda \partial \varphi)^{-1}$ denoted by $P_{\lambda, \varphi}$ is a contraction everywhere defined on $\mathbb{R}^{n}$, that is,

$$
\left\|P_{\lambda, \varphi}(x)-P_{\lambda, \varphi}(y)\right\| \leq\|x-y\|, \quad \forall x, y \in \mathbb{R}^{n}
$$

This operator $P_{\lambda, \varphi}$ is called the resolvent of order $\lambda>0$ associated to $\partial \varphi$ and for simplicity, we denote it by $P_{\varphi}$ instead of $P_{1, \varphi}$ when the parameter $\lambda=1$.

Let $\Lambda: \mathbb{R}^{n} \rightarrow \mathbb{R}^{n}$ be a continuous mapping and consider the inequality problem: Find $\bar{x} \in \mathbb{R}^{n}$ such that

$$
\langle\Lambda(\bar{x}), v-\bar{x}\rangle+\varphi(v)-\varphi(\bar{x}) \geq 0, \quad \forall v \in \mathbb{R}^{n}
$$

Clearly problem (2.1) is equivalent to the nonlinear equation: Find $\bar{x} \in \mathbb{R}^{n}$ such that

$$
\bar{x}-P_{\varphi}(\bar{x}-\Lambda(\bar{x}))=0 .
$$

In view of property $\mathrm{P} 1$ recalled earlier, it is important to compute the degree of the operator $\operatorname{id}_{\mathbb{R}^{n}}-P_{\varphi} \circ\left(\operatorname{id}_{\mathbb{R}^{n}}-\Lambda\right)$. 
Remark 2.1 If $\bar{x}$ is a solution of problem (2.1), then

$$
\langle\Lambda(\bar{x}), \xi\rangle+\varphi^{\prime}(\bar{x} ; \xi) \geq 0, \quad \forall \xi \in \mathbb{R}^{n}
$$

Indeed, let $\bar{x}$ be a solution of (2.1). Let $\xi \in \mathbb{R}^{n}$ and $\alpha>0$ be given. Setting $v=\bar{x}+\alpha \xi$ in (2.1), we get

$$
\langle\Lambda(\bar{x}), \alpha \xi\rangle+\varphi(\bar{x}+\alpha \xi)-\varphi(\bar{x}) \geq 0
$$

Thus, for all $\alpha>0$, we have

$$
\langle\Lambda(\bar{x}), \xi\rangle+\frac{\varphi(\bar{x}+\alpha \xi)-\varphi(\bar{x})}{\alpha} \geq 0
$$

Taking the limit as $\alpha \downarrow 0$ we obtain

$$
\langle\Lambda(\bar{x}), \xi\rangle+\varphi^{\prime}(\bar{x} ; \xi) \geq 0
$$

Example 2.1 Let $\varphi: \mathbb{R} \rightarrow \mathbb{R}$ be the function defined by

$$
\varphi(x)=|x|, \quad \forall x \in \mathbb{R} .
$$

We have

$$
\partial \varphi(x)= \begin{cases}1, & \text { if } x>0 \\ {[-1,1],} & \text { if } x=0 \\ -1, & \text { if } x<0\end{cases}
$$

and

$$
P_{\varphi}(x)=(I+\partial \varphi)^{-1}(x)= \begin{cases}x-1, & \text { if } x \geq 1, \\ 0, & \text { if } x \in[-1,1] \\ x+1, & \text { if } x \leq-1\end{cases}
$$

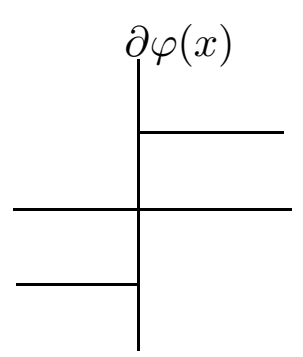

$(I+\partial \varphi)$
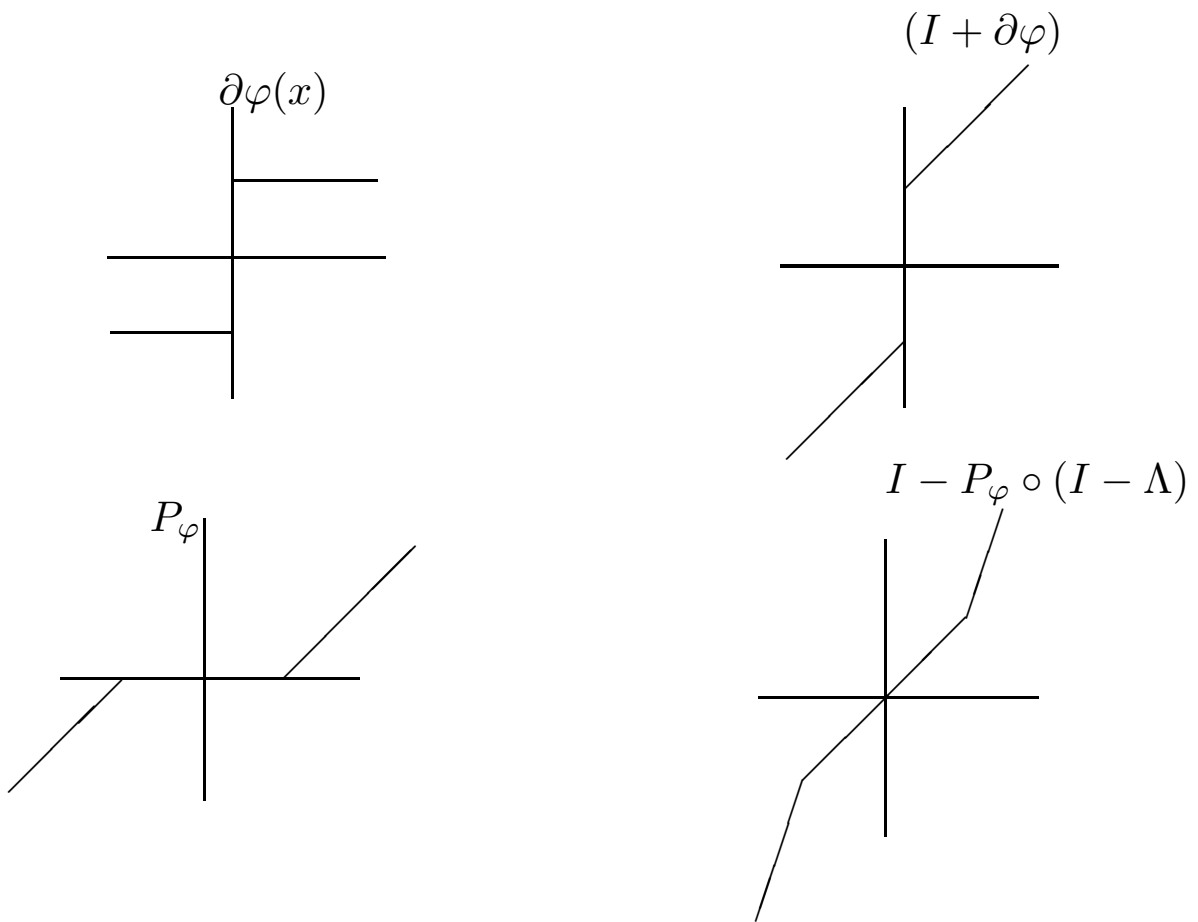

Figure 1 Example 2.1 
Setting $\Lambda(x)=2 x$, we get

$$
x-P_{\varphi}(x-\Lambda(x))= \begin{cases}x, & \text { if }|x| \leq 1 \\ 2 x-1, & \text { if } x \geq 1 \\ 2 x+1, & \text { if } x \leq-1\end{cases}
$$

We see that the operator $\operatorname{id}_{\mathbb{R}}-P_{\varphi} \circ\left(\operatorname{id}_{\mathbb{R}}-\Lambda\right)$ has a unique zero on $\mathbb{R}$.

Proposition 2.1 Let $L>0$ be given and assume that the mapping $G:[0, L] \times \mathbb{R}^{n} \rightarrow \mathbb{R}^{n}$ defined by $(\lambda, y) \mapsto G(\lambda, y)$ is continuous on $[0, L] \times \mathbb{R}^{n}$. Then, the mapping

$$
(\lambda, y) \mapsto P_{\lambda, \varphi}(G(\lambda, y))
$$

is continuous on $[0, L] \times \mathbb{R}^{n}$.

Proof Let $\left\{y_{n}\right\} \subset \mathbb{R}^{n}$ and $\left\{\lambda_{n}\right\} \subset[0, L]$ be given sequences converging respectively to $y^{*} \in \mathbb{R}^{n}$ and $\lambda_{n} \rightarrow \lambda^{*} \in \mathbb{R}$ as $n \rightarrow+\infty$. We claim that the sequence $\left\{P_{\lambda_{n}, \varphi}\left(G\left(\lambda_{n}, y_{n}\right)\right)\right\}$ tends to $P_{\lambda^{*}, \varphi}\left(G\left(\lambda^{*}, y^{*}\right)\right)$ as $n \rightarrow+\infty$. Indeed, setting $x_{n}:=P_{\lambda_{n}, \varphi}\left(G\left(\lambda_{n}, y_{n}\right)\right)$ and $x^{*}:=$ $P_{\lambda^{*}, \varphi}\left(G\left(\lambda^{*}, y^{*}\right)\right)$, we have

$$
\begin{gathered}
\left\langle x_{n}-G\left(\lambda_{n}, y_{n}\right), v-x_{n}\right\rangle+\lambda_{n} \varphi(v)-\lambda_{n} \varphi\left(x_{n}\right) \geq 0, \quad \forall v \in \mathbb{R}^{n}, \\
\left\langle x^{*}-G\left(\lambda^{*}, y^{*}\right), v-x^{*}\right\rangle+\lambda^{*} \varphi(v)-\lambda^{*} \varphi\left(x^{*}\right) \geq 0, \quad \forall v \in \mathbb{R}^{n} .
\end{gathered}
$$

Let us first check that the sequence $\left\{x_{n}\right\}$ is bounded. Indeed, suppose on the contrary that the sequence $\left\{\left\|x_{n}\right\|\right\}$ is unbounded. Setting $v:=0$ in (2.3), we obtain

$$
-\left\langle x_{n}-G\left(\lambda_{n}, y_{n}\right), x_{n}\right\rangle+\lambda_{n}\left[\varphi(0)-\varphi\left(x_{n}\right)\right] \geq 0
$$

and thus

$$
\left\|x_{n}\right\|^{2} \leq\left\|G\left(\lambda_{n}, y_{n}\right)\right\|\left\|x_{n}\right\|+\lambda_{n}\left[\varphi(0)-\varphi\left(x_{n}\right)\right] .
$$

It results that for $n$ large enough, $\left\|x_{n}\right\| \neq 0$ and

$$
1 \leq \frac{\left\|G\left(\lambda_{n}, y_{n}\right)\right\|}{\left\|x_{n}\right\|}+\frac{\lambda_{n}}{\left\|x_{n}\right\|^{2}}\left[\varphi(0)-\varphi\left(x_{n}\right)\right] .
$$

As for $n$ large enough, $\frac{1}{\left\|x_{n}\right\|} \in(0,1]$, we use the convexity of $\varphi$ to obtain

$$
\varphi\left(\frac{x_{n}}{\left\|x_{n}\right\|}\right) \leq \frac{1}{\left\|x_{n}\right\|} \varphi\left(x_{n}\right)+\left(1-\frac{1}{\left\|x_{n}\right\|}\right) \varphi(0) .
$$

Thus

$$
\frac{\varphi(0)-\varphi\left(x_{n}\right)}{\left\|x_{n}\right\|} \leq \varphi(0)-\varphi\left(\frac{x_{n}}{\left\|x_{n}\right\|}\right)
$$

From (2.5), we get

$$
1 \leq \frac{\left\|G\left(\lambda_{n}, y_{n}\right)\right\|}{\left\|x_{n}\right\|}+\lambda_{n}\left[\frac{\varphi(0)-\varphi\left(\frac{x_{n}}{\left\|x_{n}\right\|}\right)}{\left\|x_{n}\right\|}\right] .
$$

The sequence $\left\{\frac{x_{n}}{\left\|x_{n}\right\|}\right\}$ remains in the compact set $\partial \mathbb{B}_{1}$ and from the continuity of $\varphi$, we derive that the sequence $\left\{\varphi\left(\frac{x_{n}}{\left\|x_{n}\right\|}\right)\right\}$ is bounded in $\mathbb{R}$. Hence

$$
\lim _{n \rightarrow+\infty} \frac{\varphi\left(\frac{x_{n}}{\left\|x_{n}\right\|}\right)}{\left\|x_{n}\right\|}=0 .
$$


Taking now the limit as $n \rightarrow+\infty$ in (2.6), we obtain the contradiction $1 \leq 0$. The sequence $\left\{x_{n}\right\}$ is thus bounded.

Setting $v:=x^{*}$ in $(2.3)$ and $v:=x_{n}$ in $(2.4)$, we obtain the relations

$$
\begin{gathered}
\left\langle x_{n}-G\left(\lambda_{n}, y_{n}\right), x_{n}-x^{*}\right\rangle-\lambda_{n} \varphi\left(x^{*}\right)+\lambda_{n} \varphi\left(x_{n}\right) \leq 0, \\
-\left\langle x^{*}-G\left(\lambda^{*}, y^{*}\right), x_{n}-x^{*}\right\rangle-\lambda^{*} \varphi\left(x_{n}\right)+\lambda^{*} \varphi\left(x^{*}\right) \leq 0 .
\end{gathered}
$$

Thus

$$
\begin{aligned}
\left\|x_{n}-x^{*}\right\|^{2} \leq & \left\|G\left(\lambda_{n}, y_{n}\right)-G\left(\lambda^{*}, y^{*}\right)\right\|\left\|x_{n}-x^{*}\right\| \\
& +\left(\lambda_{n}-\lambda^{*}\right) \varphi\left(x^{*}\right)+\left(\lambda^{*}-\lambda_{n}\right) \varphi\left(x_{n}\right) .
\end{aligned}
$$

Using the continuity of $\varphi$ and the boundeness of $\left\{x_{n}\right\}$, we get that the sequence $\left\{\varphi\left(x_{n}\right)\right\}_{n}$ is bounded in $\mathbb{R}$. Moreover $\left\|G\left(\lambda_{n}, y_{n}\right)-G\left(\lambda^{*}, y^{*}\right)\right\| \rightarrow 0$ and $\left(\lambda_{n}-\lambda^{*}\right) \rightarrow 0$ in $\mathbb{R}$ as $n \rightarrow+\infty$. Relation (2.9) yields that $x_{n} \rightarrow x^{*}$ in $\mathbb{R}^{n}$ as $n \rightarrow+\infty$. Hence the operator $(\lambda, y) \mapsto P_{\lambda, \varphi}(G(\lambda, y))$ is continuous, which completes the proof.

Proposition 2.2 Suppose that $\Lambda: \mathbb{R}^{n} \rightarrow \mathbb{R}^{n}$ is continuous and $\varphi: \mathbb{R}^{n} \rightarrow \mathbb{R}$ is a convex function. If there exists a continuous mapping $H: \mathbb{R}^{n} \rightarrow \mathbb{R}^{n}$ and $r>0$ such that

$$
\langle\Lambda(x), H(x)\rangle+\varphi^{\prime}(x ; H(x))<0, \quad \forall x \in \partial \mathbb{B}_{r}
$$

then

$$
\operatorname{deg}\left(H, \mathbb{B}_{r}, 0\right)=(-1)^{n} \operatorname{deg}\left(\operatorname{id}_{\mathbb{R}^{n}}-P_{\varphi}\left(\operatorname{id}_{\mathbb{R}^{n}}-\Lambda\right), \mathbb{B}_{r}, 0\right)
$$

Proof Let $h:[0,1] \times \overline{\mathbb{B}}_{r} \rightarrow \mathbb{R}^{n} ;(\lambda, y) \mapsto h(\lambda, y):=y-P_{\lambda, \varphi}(y-\lambda \Lambda(y)+(1-\lambda) H(y))$. Proposition 2.1 ensures that $h$ is continuous. Let us now check that $h(\lambda, x) \neq 0, \forall x \in \partial \mathbb{B}_{r}$. Indeed, suppose on the contrary that there exists $x \in \partial \mathbb{B}_{r}$ and $\lambda \in[0,1]$ such that $h(\lambda, x)=0$, that is

$$
x=P_{\lambda, \varphi}(x-\lambda \Lambda(x)+(1-\lambda) H(x)) .
$$

We first remark that $\lambda \neq 0$. Indeed, if we suppose, on the contrary, that $\lambda=0$, then $x=$ $P_{0}(x+H(x))=x+H(x)$. This yields $H(x)=0$ which contradicts assumption $(2.10)$.

Thus $\lambda>0$ and

$$
\langle\lambda \Lambda(x)-(1-\lambda) H(x), v-x\rangle+\lambda \varphi(v)-\lambda \varphi(x) \geq 0, \quad \forall v \in \mathbb{R}^{n} .
$$

It results that (see Remark 2.1)

$$
\langle\lambda \Lambda(x)-(1-\lambda) H(x), \xi\rangle+\lambda \varphi^{\prime}(x ; \xi) \geq 0, \quad \forall \xi \in \mathbb{R}^{n}
$$

Setting $\xi:=H(x)$, we obtain

$$
\lambda\left[\langle\Lambda(x), H(x)\rangle+\varphi^{\prime}(x ; H(x))\right] \geq(1-\lambda)\|H(x)\|^{2} \geq 0,
$$

which contradicts assumption (2.10). 
Therefore,

$$
\begin{aligned}
\operatorname{deg}\left(\operatorname{id}_{\mathbb{R}^{n}}-P_{\varphi}\left(\operatorname{id}_{\mathbb{R}^{n}}-\Lambda\right), \mathbb{B}_{r}, 0\right) & =\operatorname{deg}\left(h(1, \cdot), \mathbb{B}_{r}, 0\right)=\operatorname{deg}\left(h(0, \cdot), \mathbb{B}_{r}, 0\right) \\
& =\operatorname{deg}\left(\operatorname{id}_{\mathbb{R}^{n}}-P_{0}\left(\operatorname{id}_{\mathbb{R}^{n}}+H\right), \mathbb{B}_{r}, 0\right) \\
& =\operatorname{deg}\left(-H, \mathbb{B}_{r}, 0\right)=(-1)^{n} \operatorname{deg}\left(H, \mathbb{B}_{r}, 0\right)
\end{aligned}
$$

which completes the proof.

\section{Some Existence Results for Finite Variational Inequalities}

As a direct consequence of Proposition 2.2, we have the following existence results for finite dimensional variational inequalities.

Theorem 3.1 Suppose that

(1) $\Lambda: \mathbb{R}^{n} \rightarrow \mathbb{R}^{n}$ is a continuous operator;

(2) $\varphi: \mathbb{R}^{n} \rightarrow \mathbb{R}$ is a convex function;

(3) there exists $r>0$ such that

$$
\langle\Lambda(x), x\rangle-\varphi^{\prime}(x ;-x)>0, \quad \forall x \in \partial \mathbb{B}_{r}
$$

Then there exists $\bar{x} \in \mathbb{B}_{r}$ such that

$$
\langle\Lambda(\bar{x}), v-\bar{x}\rangle+\varphi(v)-\varphi(\bar{x}) \geq 0, \quad \forall v \in \mathbb{R}^{n} .
$$

Proof Just apply Proposition 2.2 with $H:=-\mathrm{id}_{\mathbb{R}^{n}}$. Indeed, here we have

$$
\langle\Lambda(x), H(x)\rangle+\varphi^{\prime}(x ; H(x))=-\langle\Lambda(x), x\rangle+\varphi^{\prime}(x ;-x) .
$$

Theorem 3.2 Suppose that

(1) $\Lambda: \mathbb{R}^{n} \rightarrow \mathbb{R}^{n}$ is continuous;

(2) $\varphi: \mathbb{R}^{n} \rightarrow \mathbb{R}$ is convex and Lipschitz continuous with Lipschitz constant $K>0$, i.e.,

$$
|\varphi(x)-\varphi(y)| \leq K\|x-y\|, \quad \forall x, y \in \mathbb{R}^{n}
$$

(3) there exists $r>0$ such that

$$
\|\Lambda(x)\|>K, \quad \forall x \in \partial \mathbb{B}_{r} \quad \text { and } \quad \operatorname{deg}\left(\Lambda, \mathbb{B}_{r}, 0\right) \neq 0 .
$$

Then there exists $\bar{x} \in \mathbb{B}_{r}$ such that

$$
\langle\Lambda(\bar{x}), v-\bar{x}\rangle+\varphi(v)-\varphi(\bar{x}) \geq 0, \quad \forall v \in \mathbb{R}^{n}
$$

Proof Just apply Proposition 2.2 with $H:=-\Lambda$. Indeed, we have

$$
\begin{aligned}
\langle\Lambda(x), H(x)\rangle+\varphi^{\prime}(x ; H(x)) & =-\|\Lambda(x)\|^{2}+\varphi^{\prime}(x ;-\Lambda(x)) \\
& \leq-\|\Lambda(x)\|^{2}+K\|\Lambda(x)\|=\|\Lambda(x)\|(K-\|\Lambda(x)\|) .
\end{aligned}
$$

Therefore

$$
\langle\Lambda(x), H(x)\rangle+\varphi^{\prime}(x ; H(x))<0, \quad \forall x \in \partial \mathbb{B}_{r} .
$$


Proposition 2.2 ensures that

$$
\operatorname{deg}\left(\operatorname{id}_{\mathbb{R}^{n}}-P_{\varphi}\left(\operatorname{id}_{\mathbb{R}^{n}}-\Lambda\right), \mathbb{B}_{r}, 0\right)=(-1)^{n} \operatorname{deg}\left(H, \mathbb{B}_{r}, 0\right)=\operatorname{deg}\left(\Lambda, \mathbb{B}_{r}, 0\right) \neq 0
$$

Hence, there exists $\bar{x} \in \mathbb{B}_{r}$ such that $\bar{x}=P_{\varphi}(\bar{x}-\Lambda(\bar{x}))$. The conclusion follows.

Theorem 3.3 Suppose that

(1) $\Lambda: \mathbb{R}^{n} \rightarrow \mathbb{R}^{n}$ is continuous and that there exists $r>0$ such that

$$
\langle\Lambda x, x\rangle>0, \quad \forall x \in \partial \mathbb{B}_{r} \quad \text { and } \quad \operatorname{deg}\left(\operatorname{id}_{\mathbb{R}^{n}}+\Lambda, \mathbb{B}_{r}, 0\right) \neq 0
$$

(2) $\varphi: \mathbb{R}^{n} \rightarrow \mathbb{R}$ is a convex function satisfying

$$
\varphi^{\prime}(x ;-x-\Lambda x) \leq 0, \quad \forall x \in \partial \mathbb{B}_{r}
$$

Then there exists $\bar{x} \in \mathbb{B}_{r}$ such that

$$
\langle\Lambda(\bar{x}), v-\bar{x}\rangle+\varphi(v)-\varphi(\bar{x}) \geq 0, \quad \forall v \in \mathbb{R}^{n}
$$

Proof Just take $H:=-\mathrm{id}_{\mathbb{R}^{n}}-\Lambda$ in Proposition 2.2. Indeed, we have

$$
\langle\Lambda(x), H(x)\rangle+\varphi^{\prime}(x ; H(x))=-\|\Lambda(x)\|^{2}-\langle\Lambda(x), x\rangle+\varphi^{\prime}(x ;-x-\Lambda(x))<0, \quad \forall x \in \partial \mathbb{B}_{r}
$$

According to Proposition 2.2, we have

$$
\operatorname{deg}\left(\operatorname{id}_{\mathbb{R}^{n}}-P_{\varphi}\left(\operatorname{id}_{\mathbb{R}^{n}}-\Lambda\right), \mathbb{B}_{r}, 0\right)=(-1)^{n} \operatorname{deg}\left(H, \mathbb{B}_{r}, 0\right)=\operatorname{deg}\left(\operatorname{id}_{\mathbb{R}^{n}}+\Lambda, \mathbb{B}_{r}, 0\right) \neq 0
$$

Hence, there exists $\bar{x} \in \mathbb{B}_{r}$ such that $\bar{x}=P_{\varphi}(\bar{x}-\Lambda(\bar{x}))$. The conclusion follows.

Corollary 3.1 Let $f \in \mathbb{R}^{n}$ be given. Suppose that

(1) $A \in \mathbb{R}^{n \times n}$ is a real nonsingular matrix;

(2) $\varphi: \mathbb{R}^{n} \rightarrow \mathbb{R}$ is convex and Lipschitz continuous with Lipschitz constant $K>0$.

Then there exists $\bar{x} \in \mathbb{R}^{n}$ such that

$$
\langle A \bar{x}-f, v-\bar{x}\rangle+\varphi(v)-\varphi(\bar{x}) \geq 0, \quad \forall v \in \mathbb{R}^{n}
$$

Proof The result is a consequence of Theorem 3.2 with $\Lambda$ defined by

$$
\Lambda(x)=A x-f, \quad \forall x \in \mathbb{R}^{n} .
$$

The matrix $A$ is nonsingular and thus there exists $c>0$ such that $\|A x\| \geq c\|x\|, \forall x \in \mathbb{R}^{n}$. Let us choose

$$
r>\max \left\{\frac{K+\|f\|}{c},\left\|A^{-1} f\right\|\right\} .
$$

We see that if $\|x\|=r$, then

$$
\|\Lambda(x)\| \geq\|A x\|-\|f\| \geq c\|x\|-\|f\|>K .
$$

On the other hand, we remark that

$$
h(\lambda, x):=A x-\lambda f \neq 0, \quad \forall \lambda \in[0,1], x \in \partial \mathbb{B}_{r} .
$$


Indeed, suppose on the contrary that there exists $\lambda \in[0,1]$ and $x \in \partial \mathbb{B}_{r}$ such that $A x=\lambda f$. Then

$$
\|x\|=\lambda\left\|A^{-1} f\right\| \leq\left\|A^{-1} f\right\|,
$$

and we obtain the contradiction $r \leq\left\|A^{-1} f\right\|$. Thus

$\operatorname{deg}\left(A .-f, \mathbb{B}_{r}, 0\right)=\operatorname{deg}\left(h(1, \cdot), \mathbb{B}_{r}, 0\right)=\operatorname{deg}\left(h(0, \cdot), \mathbb{B}_{r}, 0\right)=\operatorname{deg}\left(A ., \mathbb{B}_{r}, 0\right)=\operatorname{sign}(\operatorname{det} A) \neq 0$, which completes the proof.

\section{The Poincaré Operator}

Let us first recall some general existence and uniqueness results (see e.g. [18]).

Theorem 4.1 Let $\varphi: \mathbb{R}^{n} \rightarrow \mathbb{R}$ be a convex function. Let $F: \mathbb{R}^{n} \rightarrow \mathbb{R}^{n}$ be a continuous operator such that for some $\omega \in \mathbb{R}, F+\omega I$ is monotone, i.e.,

$$
\langle F(x)-F(y), x-y\rangle \geq-\omega\|x-y\|^{2}, \quad \forall x, y \in \mathbb{R}^{n} .
$$

Suppose that $f:[0,+\infty) \rightarrow \mathbb{R}^{n}$ satisfies

$$
f \in C^{0}\left([0,+\infty) ; \mathbb{R}^{n}\right), \quad \frac{d f}{d t} \in L_{\mathrm{loc}}^{1}\left(0,+\infty ; \mathbb{R}^{n}\right)
$$

Let $u_{0} \in \mathbb{R}^{n}$ and $0<T<+\infty$ be given. There exists a unique $u \in C^{0}\left([0, T] ; \mathbb{R}^{n}\right)$ such that

$$
\begin{aligned}
& \frac{d u}{d t} \in L^{\infty}\left(0, T ; \mathbb{R}^{n}\right) ; \\
& u \text { is right-differentiable on }[0, T) ; \\
& u(0)=u_{0} ; \\
& \left\langle\frac{d u}{d t}(t)+F(u(t))-f(t), v-u(t)\right\rangle+\varphi(v)-\varphi(u(t)) \geq 0, \quad \forall v \in \mathbb{R}^{n}, \text { a.e., } t \in[0, T] .
\end{aligned}
$$

Remark 4.1 Suppose that $F: \mathbb{R}^{n} \rightarrow \mathbb{R}^{n}$ is of the type

$$
F(x)=A x+\Psi^{\prime}(x)+F_{1}(x), \quad \forall x \in \mathbb{R}^{n},
$$

where $A \in \mathbb{R}^{n \times n}$ is a real matrix, $\Psi \in C^{1}\left(\mathbb{R}^{n} ; \mathbb{R}\right)$ is convex and $F_{1}$ is Lipschitz continuous, i.e.,

$$
\left\|F_{1}(x)-F_{1}(y)\right\| \leq k\|x-y\|, \quad \forall x, y \in \mathbb{R}^{n}
$$

for some constant $k>0$. Then $F$ is continuous and $F+\omega I$ is monotone provided that

$$
\omega \geq \sup _{\|x\|=1}\langle-A x, x\rangle+k .
$$

We note that if $F$ is $k$-Lipschitz, then $F+k I$ is monotone.

Remark 4.2 (i) The variational inequality in (4.4) can also be written as the differential inclusion

$$
\frac{d u}{d t}(t)+F(u(t))-f(t) \in-\partial \varphi(u(t)), \quad \text { a.e., } t \in[0, T]
$$

(ii) Let $u:[0, T] \rightarrow \mathbb{R}$ be the unique solution of (4.1)-(4.4). Then

$$
\left\langle\frac{d u}{d t}(t)+F(u(t))-f(t), \xi\right\rangle+\varphi^{\prime}(u(t) ; \xi) \geq 0, \quad \forall \xi \in \mathbb{R}^{n} \text {, a.e., } t \in[0, T] \text {. }
$$


Let $T>0$ be given. Theorem 4.1 enables us to define the one parameter family $\{S(t): 0 \leq$ $t \leq T\}$ of operators from $\mathbb{R}^{n}$ into $\mathbb{R}^{n}$, as follows:

$$
\forall y \in \mathbb{R}^{n}, \quad S(t) y=u(t)
$$

$u$ being the unique solution on $[0, T]$ of the evolution problem (4.1)-(4.4). Note that

$$
\forall y \in \mathbb{R}^{n}, \quad S(0) y=y .
$$

Lemma 4.1 (See e.g. [18]) Let $T>0$ be given and let $a, b \in L^{1}(0, T ; \mathbb{R})$ with $b(t) \geq 0$ a.e., $t \in[0, T]$. Let the absolutely continuous function $w:[0, T] \rightarrow \mathbb{R}_{+}$satisfy

$$
(1-\alpha) \frac{d w}{d t}(t) \leq a(t) w(t)+b(t) w^{\alpha}(t), \quad \text { a.e., } t \in[0, T]
$$

where $0 \leq \alpha<1$. Then

$$
w^{1-\alpha}(t) \leq w^{1-\alpha}(0) e^{\int_{0}^{t} a(s) d s}+\int_{0}^{t} e^{\int_{s}^{t} a(q) d q} b(s) d s, \quad \forall t \in[0, T] .
$$

Theorem 4.2 Suppose that the assumptions of Theorem 4.1 hold. Then

$$
\|S(t) y-S(t) z\| \leq e^{\omega t}\|y-z\|, \quad \forall y, z \in \mathbb{R}^{n}, t \in[0, T] .
$$

Proof Let $y, z \in \mathbb{R}^{n}$ be given. We have

$$
\begin{gathered}
-\left\langle\frac{d}{d t} S(t) y+F(S(t) y)-f(t), S(t) z-S(t) y\right\rangle-\varphi(S(t) z)+\varphi(S(t) y) \leq 0, \quad \text { a.e., } t \in[0, T], \\
\left\langle\frac{d}{d t} S(t) z+F(S(t) z)-f(t), S(t) z-S(t) y\right\rangle-\varphi(S(t) y)+\varphi(S(t) z) \leq 0, \quad \text { a.e., } t \in[0, T] .
\end{gathered}
$$

It results that for almost every $t \in[0, T]$ we have

$$
\begin{aligned}
& \left\langle\frac{d}{d t}(S(t) z-S(t) y), S(t) z-S(t) y\right\rangle \\
\leq & \omega\|S(t) z-S(t) y\|^{-}\langle[F+\omega I](S(t) z)-[F+\omega I](S(t) y), S(t) z-S(t) y\rangle .2
\end{aligned}
$$

Since by assumption, $F+\omega I$ is monotone, it results that

$$
\frac{d}{d t}\|S(t) z-S(t) y\|^{2} \leq 2 \omega\|S(t) z-S(t) y\|^{2}, \quad \text { a.e., } t \in[0, T] .
$$

Using Lemma 4.1 with $w(\cdot):=\|S(\cdot) z-S(\cdot) y\|^{2}, a(\cdot):=2 \omega, b(\cdot)=0, \alpha=0$, we get

$$
\|S(t) z-S(t) y\|^{2} \leq\|z-y\|^{2} e^{2 \omega t}, \quad \forall t \in[0, T]
$$

The conclusion follows.

Let us now consider the Poincaré operator $S(T): \mathbb{R}^{n} \rightarrow \mathbb{R}^{n}$ given by $y \mapsto S(T) y$. Theorem 4.2 ensures that $S(T)$ is Lipschitz continuous, i.e.,

$$
\|S(T) y-S(T) z\| \leq e^{\omega T}\|y-z\|, \quad \forall y, z \in \mathbb{R}^{n}
$$


Remark 4.3 (i) Note that if $F$ is continuous and monotone, then Theorem 4.2 holds with $\omega=0$. In this case, the Poincaré operator $S(T)$ is nonexpansive, i.e.,

$$
\|S(T) y-S(T) z\| \leq\|y-z\|, \quad \forall y, z \in \mathbb{R}^{n} .
$$

(ii) If $F$ is continuous and strongly monotone, i.e., there exists $\alpha>0$ such that

$$
\langle F(x)-F(y), x-y\rangle \geq \alpha\|x-y\|^{2}, \quad \forall x, y \in \mathbb{R}^{n},
$$

then Theorem 4.2 holds with $\omega=-\alpha<0$ and the Poincaré operator $S(T)$ is a contraction.

According to (4.6), the unique solution of the problem (4.1)-(4.4) satisfies, in addition, the periodicity condition

$$
u(0)=u(T)
$$

if and only if $y$ is a fixed point of $S(T)$, that is,

$$
S(T) y=y
$$

Thus the problem of the existence of a periodic solution for the evolution problem (4.1)-(4.2), (4.4) reduces to the existence of a fixed point for $S(T)$.

\section{Periodic Solutions}

Definition 5.1 Let $\Omega \subset \mathbb{R}^{n}$ be a given subset of $\mathbb{R}^{n}$. We say that $V \in C^{1}\left(\mathbb{R}^{n} ; \mathbb{R}\right)$ is a guiding function for (4.5) on $\Omega$ provided that

$$
\langle F(x)-f(t), \nabla V(x)\rangle+\varphi^{\prime}(x ; \nabla V(x))<0, \quad \forall x \in \Omega, t \in[0, T] .
$$

Remark 5.1 (i) Suppose that there exists a guiding function $V \in C^{1}\left(\mathbb{R}^{n} ; \mathbb{R}\right)$ for $(4.5)$ on $\partial \mathbb{B}_{r}(r>0)$, i.e.,

$$
\langle F(x)-f(t), \nabla V(x)\rangle+\varphi^{\prime}(x ; \nabla V(x))<0, \quad \forall x \in \partial \mathbb{B}_{r}, t \in[0, T]
$$

Then for any $\tau \in[0, T]$, we have

$$
\operatorname{deg}\left(\nabla V, \mathbb{B}_{r}, 0\right)=(-1)^{n} \operatorname{deg}\left(\operatorname{id}_{\mathbb{R}^{n}}-P_{\varphi}\left(\operatorname{id}_{\mathbb{R}^{n}}-F+f(\tau)\right), \mathbb{B}_{r}, 0\right)
$$

Relation in (5.2) is a consequence of Proposition 2.2.

(ii) Suppose that there exists a guiding function $V \in C^{1}\left(\mathbb{R}^{n} ; \mathbb{R}\right)$ for $(4.5)$ on

$$
\Omega_{R}:=\left\{x \in \mathbb{R}^{n}:\|x\| \geq R\right\}, \quad R>0 .
$$

Then for $r \geq R$ and any $\tau \in[0, T]$, we have

$$
\operatorname{ind}(V, \infty)=(-1)^{n} \operatorname{deg}\left(\operatorname{id}_{\mathbb{R}^{n}}-P_{\varphi}\left(\operatorname{id}_{\mathbb{R}^{n}}-F+f(\tau)\right), \mathbb{B}_{r}, 0\right)
$$


Proposition 5.1 Suppose that there exists $R>0$ such that

$$
\langle F(x)-f(t), \nabla V(x)\rangle<0, \quad \forall x \in \mathbb{R}^{n},\|x\| \geq R, t \in[0, T] .
$$

Then for $r \geq R$ and any $\tau \in[0, T]$, we have

$$
\operatorname{ind}(V, \infty)=\operatorname{deg}\left(f(\tau)-F, \mathbb{B}_{r}, 0\right)
$$

Proof Let $r \geq R$ be given and let $h:[0,1] \times \overline{\mathbb{B}}_{r} \rightarrow \mathbb{R}^{n}$ be the mapping defined by $(\lambda, y) \mapsto$ $h(\lambda, y):=\lambda \nabla V(y)+(1-\lambda)(f(0)-F(y))$. We claim that $h(\lambda, y) \neq 0, \forall y \in \partial \mathbb{B}_{r}, \lambda \in[0,1]$. Indeed, suppose by contradiction that

$$
\lambda \nabla V(y)+(1-\lambda)(f(\tau)-F(y))=0
$$

for some $y \in \partial \mathbb{B}_{r}$ and $\lambda \in[0,1]$. Then

$$
\lambda\langle\nabla V(y), f(\tau)-F(y)\rangle=-(1-\lambda)\|f(\tau)-F(y)\|^{2} .
$$

If $\lambda=0$, relation (5.4) implies that $f(\tau)-F(y)=0$, and since $y \in \partial \mathbb{B}_{r}$ and $r \geq R$, we derive from relation (5.3) a contradiction. Hence, $\lambda \neq 0$ and relation (5.4) yields $\langle\nabla V(y), f(\tau)-F(y)\rangle<0$ and a contradiction to (5.3). Thus,

$$
\operatorname{deg}\left(\nabla V, \mathbb{B}_{r}, 0\right)=\operatorname{deg}\left(h(1, \cdot), \mathbb{B}_{r}, 0\right)=\operatorname{deg}\left(h(0, \cdot), \mathbb{B}_{r}, 0\right)=\operatorname{deg}\left(f(\tau)-F, \mathbb{B}_{r}, 0\right) .
$$

Theorem 5.1 Suppose that $f \in C^{0}\left([0,+\infty) ; \mathbb{R}^{n}\right)$ and $\frac{d f}{d t} \in L_{\text {loc }}^{1}\left(0,+\infty ; \mathbb{R}^{n}\right)$. Let $\varphi: \mathbb{R}^{n} \rightarrow$ $\mathbb{R}$ be a convex function. Let $F: \mathbb{R}^{n} \rightarrow \mathbb{R}^{n}$ be a mapping satisfying the conditions of Theorem 4.1. Suppose that there exist constants $c_{1}>0, c_{2}>0, c_{3}>0, C_{1} \geq 0, C_{2} \geq 0$ and a function $W \in C^{1}\left(\mathbb{R}^{n} ; \mathbb{R}\right)$ such that

$$
\begin{aligned}
c_{1}\|x\|^{2} \leq W(x) & \leq c_{3}\|x\|^{2}, \quad \forall x \in \mathbb{R}^{n}, \\
c_{2}\|\nabla W(x)\|^{2} & \leq W(x), \quad \forall x \in \mathbb{R}^{n}, \\
\langle F(x), \nabla W(x)\rangle+\varphi^{\prime}(x ; \nabla W(x)) & \leq C_{1}\|\nabla W(x)\|^{2}+C_{2}\|\nabla W(x)\|, \quad \forall x \in \mathbb{R}^{n} .
\end{aligned}
$$

Let $T>0$ be given. Assume that there exists a (guiding) function $V \in C^{1}\left(\mathbb{R}^{n} ; \mathbb{R}\right)$ and $R>0$ such that

$$
\langle F(x)-f(t), \nabla V(x)\rangle+\varphi^{\prime}(x ; \nabla V(x))<0, \quad \forall x \in \mathbb{R}^{n},\|x\| \geq R, t \in[0, T] .
$$

Then there exists $r_{0}>R$ such that for any $\tau \in[0, T]$, we have

$$
\begin{aligned}
\operatorname{deg}\left(\operatorname{id}_{\mathbb{R}^{n}}-S(T)(\cdot), \mathbb{B}_{r}, 0\right) & =\operatorname{deg}\left(\operatorname{id}_{\mathbb{R}^{n}}-P_{\varphi}\left(\operatorname{id}_{\mathbb{R}^{n}}-F+f(\tau)\right), \mathbb{B}_{r}, 0\right) \\
& =(-1)^{n} \operatorname{ind}(V, \infty), \quad \forall r \geq r_{0}
\end{aligned}
$$

Proof Let us first remark that without any loss of generality, we may assume that $C_{1}>0$. We set

$$
r_{0}:=R \frac{\sqrt{c_{3}}}{\sqrt{c_{1}}} e^{\frac{C_{1}}{2 c_{2}} T}+\frac{\sqrt{c_{2}} C_{2}}{\sqrt{c_{1}} C_{1}}\left(e^{\frac{C_{1}}{2 c_{2}} T}-1\right)+\frac{1}{2 \sqrt{c_{1} c_{2}}} \int_{0}^{T}\|f(s)\| e^{\frac{C_{1}}{2 c_{2}} s} d s .
$$


(1) We claim that if $y \in \mathbb{R}^{n},\|y\|=r$ with $r \geq r_{0}$, then

$$
\|S(t) y\| \geq R, \quad \forall t \in[0, T]
$$

Suppose by contradiction that there exists $t^{*} \in[0, T]$ such that $\left\|S\left(t^{*}\right) y\right\|<R$. We know that $u(\cdot) \equiv S(\cdot) y$ satisfies $(4.5)$, i.e.,

$$
\frac{d u}{d t}(t)+F(u(t))-f(t) \in-\partial \varphi(u(t)), \quad \text { a.e., } t \in[0, T]
$$

and thus

$$
\frac{d u}{d t}\left(t^{*}-t\right)+F\left(u\left(t^{*}-t\right)\right)-f\left(t^{*}-t\right) \in-\partial \varphi\left(u\left(t^{*}-t\right)\right), \quad \text { a.e., } t \in\left[0, t^{*}\right] .
$$

Setting

$$
Y(t)=u\left(t^{*}-t\right), \quad t \in\left[0, t^{*}\right]
$$

we derive

$$
-\frac{d Y}{d t}(t)+F(Y(t))-f\left(t^{*}-t\right) \in-\partial \varphi(Y(t)), \quad \text { a.e., } t \in\left[0, t^{*}\right] .
$$

Thus, for every $v \in \mathbb{R}^{n}$ and almost every $t \in\left[0, t^{*}\right]$, we have

$$
\varphi(v)-\varphi(Y(t))+\left\langle-\frac{d Y}{d t}(t), v-Y(t)\right\rangle \geq\left\langle-F(Y(t))+f\left(t^{*}-t\right), v-Y(t)\right\rangle .
$$

Hence

$$
\left\langle\frac{d Y}{d t}(t), \xi\right\rangle \leq\left\langle F(Y(t))-f\left(t^{*}-t\right), \xi\right\rangle+\varphi^{\prime}(Y(t) ; \xi), \quad \forall \xi \in \mathbb{R}^{n}, \text { a.e., } t \in\left[0, t^{*}\right] .
$$

Set $v=\nabla W(Y(t))$ to obtain

$$
\begin{aligned}
& \left\langle\frac{d Y}{d t}(t), \nabla W(Y(t))\right\rangle \\
\leq & \left\langle F(Y(t))-f\left(t^{*}-t\right), \nabla W(Y(t))\right\rangle+\varphi^{\prime}(Y(t) ; \nabla W(Y(t))) \\
\leq & C_{1}\|\nabla W(Y(t))\|^{2}+C_{2}\|\nabla W(Y(t))\|+\left\|f\left(t^{*}-t\right)\right\|\|\nabla W(Y(t))\| \\
\leq & \frac{C_{1}}{c_{2}} W(Y(t))+\frac{C_{2}}{\sqrt{c_{2}}} \sqrt{W(Y(t))}+\frac{1}{\sqrt{c_{2}}}\left\|f\left(t^{*}-t\right)\right\| \sqrt{W(Y(t))}, \quad \text { a.e., } t \in\left[0, t^{*}\right] .
\end{aligned}
$$

Thus

$\frac{1}{2} \frac{d}{d t} W(Y(t)) \leq \frac{C_{1}}{2 c_{2}} W(Y(t))+\frac{C_{2}}{2 \sqrt{c_{2}}} \sqrt{W(Y(t))}+\frac{1}{2 \sqrt{c_{2}}}\left\|f\left(t^{*}-t\right)\right\| \sqrt{W(Y(t))}, \quad$ a.e., $t \in\left[0, t^{*}\right]$.

Using Lemma 4.1 with $w(\cdot):=W(Y(\cdot)), a(\cdot):=\frac{C_{1}}{2 c_{2}}, b(\cdot):=\frac{1}{2 \sqrt{c_{2}}}\left(C_{2}+\left\|f\left(t^{*}-\cdot\right)\right\|\right)$ and $\alpha:=\frac{1}{2}$, we obtain for every $t \in\left[0, t^{*}\right]$,

$$
\sqrt{W(Y(t))} \leq \sqrt{W(Y(0))} e^{\frac{C_{1}}{2 c_{2}} t}+\int_{0}^{t} \frac{C_{2}}{2 \sqrt{c_{2}}} e^{\frac{C_{1}}{2 c_{2}}(t-s)} d s+\int_{0}^{t} \frac{1}{2 \sqrt{c_{2}}}\left\|f\left(t^{*}-s\right)\right\| e^{\frac{C_{1}}{2 c_{2}}(t-s)} d s .
$$

Thus, for every $t \in\left[0, t^{*}\right]$,

$$
\|Y(t)\| \leq \frac{1}{\sqrt{c_{1}}}\left(\sqrt{c_{3}}\|Y(0)\| e^{\frac{C_{1}}{2 c_{2}} t}+\int_{0}^{t} \frac{C_{2}}{2 \sqrt{c_{2}}} e^{\frac{C_{1}}{2 c_{2}}(t-s)} d s+\int_{0}^{t} \frac{1}{2 \sqrt{c_{2}}}\left\|f\left(t^{*}-s\right)\right\| e^{\frac{C_{1}}{2 c_{2}}(t-s)} d s\right) .
$$


Since $Y\left(t^{*}\right)=u(0)=S(0) y=y$ and $Y(0)=u\left(t^{*}\right)=S\left(t^{*}\right) y$, we get

$$
\begin{aligned}
\|y\| & \leq \frac{1}{\sqrt{c_{1}}}\left(\sqrt{c_{3}}\left\|S\left(t^{*}\right) y\right\| e^{\frac{C_{1}}{2 c_{2}} t^{*}}+\int_{0}^{t^{*}} \frac{C_{2}}{2 \sqrt{c_{2}}} e^{\frac{C_{1}}{2 c_{2}}\left(t^{*}-s\right)} d s+\int_{0}^{t^{*}} \frac{1}{2 \sqrt{c_{2}}}\left\|f\left(t^{*}-s\right)\right\| e^{\frac{C_{1}}{2 c_{2}}\left(t^{*}-s\right)} d s\right) \\
& <R \frac{\sqrt{c_{3}}}{\sqrt{c_{1}}} e^{\frac{C_{1}}{2 c_{2}} T}+\frac{\sqrt{c_{2}} C_{2}}{\sqrt{c_{1}} C_{1}}\left(e^{\frac{C_{1}}{2 c_{2}} T}-1\right)+\frac{1}{2 \sqrt{c_{1} c_{2}}} \int_{0}^{T}\|f(s)\| e^{\frac{C_{1}}{2 c_{2}} s} d s=r_{0} .
\end{aligned}
$$

Hence, $\|y\|<r_{0}$, a contradiction.

Let $r \geq r_{0}$ be given.

(2) We claim that there exists $\varepsilon>0$ and $T^{*} \in(0, T]$ such that

$\langle F(x)-f(t), \nabla V(y)\rangle+\varphi^{\prime}(x ; \nabla V(y))<0, \quad \forall x \in \mathbb{R}^{n}, y \in \mathbb{R}^{n},\|y\|=r,\|x-y\| \leq \varepsilon, t \in\left[0, T^{*}\right]$.

Indeed, recalling that the mapping $(z, \xi) \mapsto \varphi^{\prime}(z ; \xi)$ is upper semicontinuous (see e.g. [11]), we note that the mapping $(t, x, y) \mapsto\langle F(x)-f(t), \nabla V(y)\rangle+\varphi^{\prime}(x ; \nabla V(y))$ is upper semicontinuous on $[0, T] \times \mathbb{R}^{n} \times \mathbb{R}^{n}$, and if $y \in \mathbb{R}^{n},\|y\|=r \geq r_{0} \geq R$, then (by condition (5.8))

$$
\langle F(y)-f(0), \nabla V(y)\rangle+\varphi^{\prime}(y ; \nabla V(y))<0 .
$$

Thus, for $t>0$ close to 0 , say $t \leq T^{*}$, and $x$ closed to $y$, say $\|x-y\| \leq \varepsilon, \varepsilon>0$ small enough, we have

$$
\langle F(x)-f(0), \nabla V(y)\rangle+\varphi^{\prime}(x ; \nabla V(y))<0 .
$$

(3) We claim that there exists $\bar{T} \in\left(0, T^{*}\right]$ such that

$$
\|S(t) y-y\| \leq \varepsilon, \quad \forall y \in \partial \mathbb{B}_{r}, \forall t \in[0, \bar{T}]
$$

Indeed, by contradiction suppose that there exists sequences $\left\{t_{n}\right\}$ and $\left\{y_{n}\right\}$ with $t_{n} \in\left[0, \frac{T^{*}}{n}\right]$ $(n \in \mathbb{N}, n \geq 1),\left\|y_{n}\right\|=r$ and such that $\left\|S\left(t_{n}\right) y_{n}-y_{n}\right\|>\varepsilon$. Taking a subsequence, $y$, if necessary, we may assume that $t_{n} \rightarrow 0+$ and $y_{n} \rightarrow y^{*} \in \partial \mathbb{B}_{r}$. On the other hand, we have

$$
\begin{aligned}
\left\|S\left(t_{n}\right) y_{n}-y_{n}\right\| & =\left\|S\left(t_{n}\right) y_{n}-S\left(t_{n}\right) y^{*}+S\left(t_{n}\right) y^{*}-y_{n}\right\| \\
& \leq\left\|S\left(t_{n}\right) y_{n}-S\left(t_{n}\right) y^{*}\right\|+\left\|S\left(t_{n}\right) y^{*}-y_{n}\right\| .
\end{aligned}
$$

Then, using Theorem 4.2, we obtain

$$
\left\|S\left(t_{n}\right) y_{n}-y_{n}\right\| \leq \sqrt{e^{2 w t_{n}}}\left\|y_{n}-y^{*}\right\|+\left\|S\left(t_{n}\right) y^{*}-y_{n}\right\|
$$

Using the continuity of the map $t \mapsto S(t) y$, we see that $\left\|S\left(t_{n}\right) y_{n}-y_{n}\right\| \rightarrow 0$, a contradiction.

(4) Let $H_{\bar{T}}:[0,1] \times \bar{C}_{r} \rightarrow \mathbb{R}^{n} ;(\lambda, y) \rightarrow H_{\bar{T}}(\lambda, y):=y-(1-\lambda) \nabla V(y)-S(\lambda \bar{T}) y$. We claim that the homotopy $H_{\bar{T}}$ is such that $0 \neq H_{\bar{T}}(\lambda, y), \forall y \in \partial \mathbb{B}_{r}, \lambda \in[0,1]$. By contradiction, suppose that there exists $y \in \mathbb{R}^{n},\|y\|=r$ and $\lambda \in[0,1]$ such that

$$
y-(1-\lambda) \nabla V(y)-S(\lambda \bar{T}) y=0 .
$$

Then

$$
S(\lambda \bar{T}) y-y=-(1-\lambda) \nabla V(y)
$$


and thus

$$
\langle S(\lambda \bar{T}) y-y, \nabla V(y)\rangle=-(1-\lambda)\|\nabla V(y)\|^{2} \leq 0 .
$$

On the other hand, we know that

$$
\begin{array}{r}
\left\langle\frac{d}{d t} S(t) y, v-S(t) y\right\rangle+\varphi(v)-\varphi(S(t) y) \geq\langle-F(S(t) y)+f(t), v-S(t) y\rangle, \\
\forall v \in \mathbb{R}^{n}, \text { a.e., } t \in[0, T] .
\end{array}
$$

Thus

$$
\left\langle\frac{d}{d t} S(t) y, \nabla V(y)\right\rangle+\varphi^{\prime}(S(t) y ; \nabla V(y)) \geq\langle-F(S(t) y)+f(t), \nabla V(y)\rangle, \quad \text { a.e., } t \in[0, T] .
$$

Therefore

$$
\left\langle\int_{0}^{\lambda \bar{T}} \frac{d}{d s} S(s) y d s, \nabla V(y)\right\rangle \geq \int_{0}^{\lambda \bar{T}}\langle-F(S(s) y)+f(s), \nabla V(y)\rangle-\varphi^{\prime}(S(s) y ; \nabla V(y)) d s .
$$

Part (1) of this proof ensures that $\|S(t) y\| \geq R, \forall t \in[0, \lambda \bar{T}] \subset[0, T]$. Part (3) of this proof guarantees that $\|S(t) y-y\| \leq \varepsilon, \forall t \in[0, \lambda \bar{T}] \subset[0, \bar{T}]$. Then using part (2) of this proof, we may assert that the map $s \mapsto\langle F(S(s) y)-f(s), \nabla V(y)\rangle+\varphi^{\prime}(S(s) y ; \nabla V(y))$ is upper semicontinuous and strictly negative on $[0, \lambda \bar{T}]$. Thus

$$
\int_{0}^{\lambda \bar{T}}\langle-F(S(s) y)+f(s), \nabla V(y)\rangle-\varphi^{\prime}(S(s) y ; \nabla V(y)) d s>0
$$

and we obtain

$$
\langle S(\lambda \bar{T}) y-y, \nabla V(y)\rangle=\left\langle\int_{0}^{\lambda \bar{T}} \frac{d}{d s} S(s) y d s, \nabla V(y)\right\rangle>0 .
$$

This contradicts relation $(5.12)$.

(5) Thanks to part (4) of this proof, we may use the invariance by homotopy property of the topological degree and observe that

$$
\begin{aligned}
\operatorname{deg}\left(\operatorname{id}_{\mathbb{R}^{n}}-S(\bar{T}) ., \mathbb{B}_{r}, 0\right) & =\operatorname{deg}\left(H_{\bar{T}}(1, \cdot), \mathbb{B}_{r}, 0\right)=\operatorname{deg}\left(H_{\bar{T}}(0, \cdot), \mathbb{B}_{r}, 0\right) \\
& =\operatorname{deg}\left(-\nabla V, \mathbb{B}_{r}, 0\right)=(-1)^{n} \operatorname{deg}\left(\nabla V, \mathbb{B}_{r}, 0\right)
\end{aligned}
$$

(6) Let $H:[0,1] \times \overline{\mathbb{B}}_{r} \rightarrow \mathbb{R}^{n} ;(\lambda, y) \rightarrow H(\lambda, y):=y-S((1-\lambda) T+\lambda \bar{T}) y$. We claim that $H(\lambda, y) \neq 0, \forall y \in \partial \mathbb{B}_{r}, \lambda \in[0,1]$. By contradiction, suppose that there exists $y \in \mathbb{R}^{n},\|y\|=r$ and $\lambda \in[0,1]$ such that $y=S((1-\lambda) T+\lambda \bar{T}) y$. Let us set $h:=(1-\lambda) T+\lambda \bar{T}$. We have

$$
y=S(h) y .
$$

Thus

$$
V(y)=V(S(h) y) .
$$

On the other hand,

$$
\begin{array}{r}
\left\langle\frac{d}{d t} S(t) y, v-S(t) y\right\rangle+\varphi(v)-\varphi(S(t) y) \geq\langle-F(S(t) y)+f(t), v-S(t) y\rangle, \\
\forall v \in \mathbb{R}^{n}, \text { a.e., } t \in[0, T] .
\end{array}
$$


Thus

$$
\begin{array}{r}
\left\langle\frac{d}{d t} S(t) y, \nabla V(S(t) y)\right\rangle+\varphi^{\prime}(S(t) y ; \nabla V(S(t) y)) \geq\langle-F(S(t) y)+f(t), \nabla V(S(t) y)\rangle \\
\text { a.e., } t \in[0, T] .
\end{array}
$$

Part (1) of this proof ensures that $\|S(t) y\| \geq R, \forall t \in[0, T]$. The map $s \mapsto\langle F(S(s) y)-$ $f(s), \nabla V(S(s) y)\rangle+\varphi^{\prime}(S(s) y ; \nabla V(S(s) y))$ is upper semicontinuous and (by condition (5.8)) strictly negative on $[0, T]$. Thus, using (5.16), we obtain

$$
\begin{aligned}
V(S(h) y)-V(y) & =\int_{0}^{h} \frac{d}{d s} V(S(s) y) d s=\int_{0}^{h}\left\langle\frac{d}{d s} S(s) y, \nabla V(S(s) y)\right\rangle \\
& \geq \int_{0}^{h}\langle-F(S(s) y)+f(s), \nabla V(S(s) y)\rangle-\varphi^{\prime}(S(s) y ; \nabla V(S(s) y)) d s>0 .
\end{aligned}
$$

This contradicts (5.14).

(7) Thanks to part (6) of this proof, we may use the invariance by homotopy property of the topological degree and see that

$\operatorname{deg}\left(\operatorname{id}_{\mathbb{R}^{n}}-S(T) ., \mathbb{B}_{r}, 0\right)=\operatorname{deg}\left(H(0, \cdot), \mathbb{B}_{r}, 0\right)=\operatorname{deg}\left(H(1, \cdot), \mathbb{B}_{r}, 0\right)=\operatorname{deg}\left(\operatorname{id}_{\mathbb{R}^{n}}-S(\bar{T}) ., \mathbb{B}_{r}, 0\right)$.

In conclusion, for all $r \geq r_{0}$, we have

$$
\begin{aligned}
& \operatorname{deg}\left(\operatorname{id}_{\mathbb{R}^{n}}-S(T) ., \mathbb{B}_{r}, 0\right)=\operatorname{deg}\left(\operatorname{id}_{\mathbb{R}^{n}}-S(\bar{T}) ., \mathbb{B}_{r}, 0\right), \\
& \operatorname{deg}\left(\operatorname{id}_{\mathbb{R}^{n}}-S(\bar{T}) ., \mathbb{B}_{r}, 0\right)=(-1)^{n} \operatorname{deg}\left(\nabla V, \mathbb{B}_{r}, 0\right) .
\end{aligned}
$$

Thus

$$
\operatorname{deg}\left(\operatorname{id}_{\mathbb{R}^{n}}-S(T) ., \mathbb{B}_{r}, 0\right)=(-1)^{n} \operatorname{ind}(V, \infty) .
$$

Finally, for any $\tau \in[0, T]$, we have also (see Proposition 2.2 and Remark 5.1)

$$
(-1)^{n} \operatorname{ind}(V, \infty)=\operatorname{deg}\left(\operatorname{id}_{\mathbb{R}^{n}}-P_{\varphi}\left(\operatorname{id}_{\mathbb{R}^{n}}-F+f(\tau)\right), \mathbb{B}_{r}, 0\right) .
$$

Corollary 5.1 Suppose that $f \in C^{0}\left([0,+\infty) ; \mathbb{R}^{n}\right)$ and $\frac{d f}{d t} \in L_{\mathrm{loc}}^{1}\left(0,+\infty ; \mathbb{R}^{n}\right)$. Let $\varphi: \mathbb{R}^{n} \rightarrow$ $\mathbb{R}$ be a convex function. Let $F: \mathbb{R}^{n} \rightarrow \mathbb{R}^{n}$ be a mapping satisfying the conditions of Theorem 4.1. Suppose in addition that there exist constants $C_{1} \geq 0, C_{2} \geq 0$ such that

$$
\langle F(x), x\rangle+\varphi^{\prime}(x ; x) \leq C_{1}\|x\|^{2}+C_{2}\|x\|, \quad \forall x \in \mathbb{R}^{n} .
$$

Let $T>0$ be given. Assume that there exists $V \in C^{1}\left(\mathbb{R}^{n} ; \mathbb{R}\right)$ and $R>0$ such that

$$
\langle F(x)-f(t), \nabla V(x)\rangle+\varphi^{\prime}(x ; \nabla V(x))<0, \quad \forall x \in \mathbb{R}^{n},\|x\| \geq R, t \in[0, T],
$$

and

$$
\operatorname{ind}(V, \infty) \neq 0 \text {. }
$$

Then there exists at least one $u \in C^{0}\left([0, T] ; \mathbb{R}^{n}\right)$ such that $\frac{d u}{d t} \in L^{\infty}\left(0, T ; \mathbb{R}^{n}\right)$,

$$
\begin{aligned}
& u(0)=u(T) \\
& \left\langle\frac{d u}{d t}(t)+F(u(t))-f(t), v-u(t)\right\rangle+\varphi(v)-\varphi(u(t)) \geq 0, \quad \forall v \in \mathbb{R}^{n}, \text { a.e., } t \in[0, T] .
\end{aligned}
$$


Proof We may apply Theorem 5.1 with $W(x)=\frac{1}{2}\|x\|^{2}$. It results that for $r>0$ large enough, we have $\operatorname{deg}\left(\mathrm{id}_{\mathbb{R}^{n}}-S(T), \mathbb{B}_{r}, 0\right) \neq 0$ and the existence of a fixed point for the Poincaré operator follows from the existence property of the topological degree.

Corollary 5.2 Suppose that $f \in C^{0}\left([0,+\infty) ; \mathbb{R}^{n}\right)$ and $\frac{d f}{d t} \in L_{\mathrm{loc}}^{1}\left(0,+\infty ; \mathbb{R}^{n}\right)$. Let $\varphi: \mathbb{R}^{n} \rightarrow$ $\mathbb{R}$ be a convex and Lipschitz continuous function. Let $F: \mathbb{R}^{n} \rightarrow \mathbb{R}^{n}$ be a mapping satisfying the conditions of Theorem 4.1. Suppose in addition that there exists $\Phi \in C^{1}\left(\mathbb{R}^{n} ; \mathbb{R}\right)$ such that

$$
\begin{aligned}
& \operatorname{ind}(\Phi, \infty) \neq 0, \\
& F(x)=\nabla \Phi(x), \quad \forall x \in \mathbb{R}^{n},
\end{aligned}
$$

and

$$
c_{1}\|x\|^{2} \leq c_{2}\|\nabla \Phi(x)\|^{2} \leq \Phi(x) \leq c_{3}\|x\|^{2}, \quad \forall x \in \mathbb{R}^{n}
$$

for some constants $c_{1}>0, c_{2}>0$ and $c_{3}>0$.

Let $T>0$ be given. There exists at least one $u \in C^{0}\left([0, T] ; \mathbb{R}^{n}\right)$ such that $\frac{d u}{d t} \in L^{\infty}\left(0, T ; \mathbb{R}^{n}\right)$,

$$
\begin{aligned}
& u(0)=u(T) \\
& \left\langle\frac{d u}{d t}(t)+F(u(t))-f(t), v-u(t)\right\rangle+\varphi(v)-\varphi(u(t)) \geq 0, \quad \forall v \in \mathbb{R}^{n}, \text { a.e., } t \in[0, T] .
\end{aligned}
$$

Proof We may apply Theorem 5.1 with $W=\Phi$ and $V=-\Phi$. Indeed, let $K>0$ denote the Lipschitz constant of $\varphi$. We have

$\langle F(x), W(x)\rangle+\varphi^{\prime}(x ; W(x))=\|\nabla \Phi(x)\|^{2}+\varphi^{\prime}(x ; \nabla \Phi(x)) \leq\|\nabla \Phi(x)\|^{2}+K\|\nabla \Phi(x)\|, \quad \forall x \in \mathbb{R}^{n}$.

Moreover, let $C:=K+\max _{t \in[0, T]}\|f(t)\|$ and $R>\frac{C}{c_{1}} \sqrt{\frac{c_{3}}{c_{2}}}$. We have

$$
\begin{aligned}
& \langle F(x)-f(t), V(x)\rangle+\varphi^{\prime}(x ; V(x)) \\
= & -\|\nabla \Phi(x)\|^{2}+\langle f(t), \nabla \Phi(x)\rangle+\varphi^{\prime}(x ;-\nabla \Phi(x)) \\
\leq & -\|\nabla \Phi(x)\|^{2}+C\|\nabla \Phi(x)\| \leq-c_{1}\|x\|^{2}+C \sqrt{\frac{c_{3}}{c_{2}}}\|x\| \\
\leq & \|x\|\left(C \sqrt{\frac{c_{3}}{c_{2}}}-c_{1}\|x\|\right)<0, \quad \forall x \in \mathbb{R}^{n},\|x\| \geq R, t \in[0, T] .
\end{aligned}
$$

Corollary 5.3 Suppose that $f \in C^{0}\left([0,+\infty) ; \mathbb{R}^{n}\right)$ and $\frac{d f}{d t} \in L_{\text {loc }}^{1}\left(0,+\infty ; \mathbb{R}^{n}\right)$. Let $\varphi: \mathbb{R}^{n} \rightarrow$ $\mathbb{R}$ be a convex and Lipschitz continuous function and let $T>0$ be given. Let $A \in \mathbb{R}^{n \times n}$ be a real matrix and denote by $\sigma(A)$ the set of eigenvalues of $A$. If

$$
\operatorname{Re}(\lambda)>0, \quad \forall \lambda \in \sigma(A)
$$

then there exists at least one $u \in C^{0}\left([0, T] ; \mathbb{R}^{n}\right)$ such that $\frac{d u}{d t} \in L^{\infty}\left(0, T ; \mathbb{R}^{n}\right)$,

$$
\begin{aligned}
& u(0)=u(T), \\
& \left\langle\frac{d u}{d t}(t)+A u(t)-f(t), v-u(t)\right\rangle+\varphi(v)-\varphi(u(t)) \geq 0, \quad \forall v \in \mathbb{R}^{n}, \text { a.e., } t \in[0, T] .
\end{aligned}
$$


Proof Our assumption $\operatorname{Re}(\sigma(A)) \subset] 0,+\infty[$ together with Lyapunov's Theorem ensures the existence of a positive definite matrix $G$ such that

$$
G A+A^{T} G=I
$$

Let us now define $V \in C^{1}\left(\mathbb{R}^{n} ; \mathbb{R}\right)$ by

$$
V(x):=-\frac{1}{2}\left\langle\left(G+G^{T}\right) x, x\right\rangle, \quad \forall x \in \mathbb{R}^{n} .
$$

Then

$$
\begin{aligned}
& \nabla V(x)=-\left(G+G^{T}\right) x \\
& \langle A x, \nabla V(x)\rangle=-\langle A x, G x\rangle-\left\langle A x, G^{T} x\right\rangle=-\left\langle x, A^{T} G x\right\rangle-\langle G A x, x\rangle=-\|x\|^{2} .
\end{aligned}
$$

If we set $C=\left(\max _{s \in[0, T]}\|f(s)\|+K\right)\left\|G+G^{T}\right\|$ and if $K$ denotes the Lipschitz constant of $\varphi$, then we have

$$
\langle A x-f(t), \nabla V(x)\rangle+\varphi^{\prime}(x ; \nabla V(x)) \leq-\|x\|^{2}+C\|x\|, \quad \forall x \in \mathbb{R}^{n}, t \in[0, T] .
$$

Thus, for $R>0$ large enough, condition (5.18) is satisfied. It is also clear that condition (5.17) holds (see Remark 5.2) and that all assumptions of Theorem 4.1 are satisfied. Moreover

$$
\operatorname{ind}(V, \infty)=\operatorname{ind}\left(G+G^{T}, \infty\right)=\operatorname{sign} \operatorname{det}\left(G+G^{T}\right) \neq 0
$$

since $G+G^{T}$ is positive definite and thus nonsingular.

The conclusion follows from Corollary 5.1.

Remark 5.2 Suppose that $\varphi: \mathbb{R}^{n} \rightarrow \mathbb{R}$ is Lipschitz continuous with Lipschitz constant $K$. Then

(i) If $F$ has a linear growth, i.e., there exist $c_{1} \geq 0$ and $c_{2} \geq 0$ such that

$$
\|F(x)\| \leq c_{1}\|x\|+c_{2}, \quad \forall x \in \mathbb{R}^{n},
$$

then condition (5.17) of Corollary 5.1 holds with $C_{1}=c_{1}$ and $C_{2}=c_{2}+K$.

(ii) If $\langle F(x), x\rangle \leq 0, \forall x \in \mathbb{R}^{n}$, then condition (5.17) of Corollary 5.1 holds with $C_{1}=0$ and $C_{2}=K$.

Example 5.1 Let $F: \mathbb{R}^{n} \rightarrow \mathbb{R}^{n}$ and $\varphi: \mathbb{R}^{n} \rightarrow \mathbb{R}$ defined by

$$
F(x)=x \quad \text { and } \quad \varphi(x)=\|x\|, \quad x \in \mathbb{R}^{n} .
$$

We have

$$
\langle F(x), x\rangle+\varphi^{\prime}(x ; x) \leq\|x\|^{2}+\|x\| .
$$

Hence Condition (5.17) of Corollary 5.1 is satisfied with $C_{1}=C_{2}=1$.

Example 5.2 If we take $F(x)=-x$ and $\varphi(x)=\|x\|$, then

$$
\langle F(x), x\rangle+\varphi^{\prime}(x ; x) \leq-\|x\|^{2}+\|x\| \leq\|x\| .
$$

Hence Condition (5.17) of Corollary 5.1 is satisfied with $C_{1}=0$ and $C_{2}=1$. 


\section{Second Order Periodic Dynamical System with Friction}

Let us consider the following second order dynamical system with periodic conditions: For $\left(q_{0}, \dot{q}_{0}\right) \in \mathbb{R}^{m} \times \mathbb{R}^{m}$, we consider the problem $P\left(q_{0}, \dot{q}_{0}\right)$ of finding a function $t \mapsto q(t)(t \in[0, T])$ with $q \in C^{1}\left([0, T] ; \mathbb{R}^{m}\right)$, such that

$$
\begin{aligned}
& \frac{d^{2} q}{d t^{2}} \in L^{\infty}\left(0, T ; \mathbb{R}^{m}\right), \\
& \frac{d q}{d t} \text { is right-differentiable on }[0, T], \\
& q(0)=q(T) \text { and } \dot{q}(0)=\dot{q}(T), \\
& M \frac{d^{2} q}{d t^{2}}(t)+C \frac{d q}{d t}(t)+K(q(t))-F(t) \in-H_{1} \partial \Phi\left(H_{1}^{T} \frac{d q}{d t}(t)\right), \quad \text { a.e., } t \in[0, T] .
\end{aligned}
$$

In this problem $\Phi: \mathbb{R}^{l} \rightarrow \mathbb{R}$ is a convex function, $M \in \mathbb{R}^{m \times m}$ is a symmetric and positive definite matrix, $C \in \mathbb{R}^{m \times m}$ and $K \in \mathbb{R}^{m \times m}$ are given matrices and $H_{1} \in \mathbb{R}^{m \times l}$ is a given matrix whose coefficients are related to the directions of friction forces. The function $F \in C^{0}\left([0,+\infty) ; \mathbb{R}^{m}\right)$ is such that $\frac{d F}{d t} \in L_{\mathrm{loc}}^{1}\left([0,+\infty) ; \mathbb{R}^{m}\right)$.

The second order dynamical system (6.4) is useful for the study of many problems in unilateral mechanics. Indeed, the motion of various mechanical systems with frictional contact can be studied within the framework of equation (6.4). For such problems $m$ is the number of degrees of freedom, $M$ is the mass matrix, $C$ is the viscous damping matrix and $K$ is the stiffness matrix. The vector $q \in \mathbb{R}^{m}$ is the vector of generalised coordinates. The term $H_{1} \partial \Phi\left(H_{1}^{T} \cdot\right)$ is used to model the unilaterality of the contact induced by the friction forces.

Since the matrix $M$ is symmetric and positive definite, then problem (6.4) is equivalent to the first order variational inclusion:

$$
\left\{\begin{array}{l}
\dot{x}(t)+A(x(t))-f(t) \in-\partial \varphi(x(t)), \\
x(0)=x(T)
\end{array}\right.
$$

where the vector $x=\left(\begin{array}{l}x_{1} \\ x_{2}\end{array}\right) \in \mathbb{R}^{n}(n=2 m)$ and the matrix $A \in \mathbb{R}^{n \times n}$ is defined by

$$
A=\left(\begin{array}{cc}
0_{m \times m} & -I_{m \times m} \\
M^{-\frac{1}{2}} K M^{-\frac{1}{2}} & M^{-\frac{1}{2}} C M^{-\frac{1}{2}}
\end{array}\right)
$$

with

$$
\begin{aligned}
& x(0)=\left(\begin{array}{c}
M^{-\frac{1}{2}} q(0) \\
M^{-\frac{1}{2}} \dot{q}(0)
\end{array}\right), \quad x(T)=\left(\begin{array}{c}
M^{-\frac{1}{2}} q(T) \\
M^{-\frac{1}{2}} \dot{q}(T)
\end{array}\right), \\
& f(t)=\left(\begin{array}{c}
0_{m \times m} \\
F(t)
\end{array}\right)
\end{aligned}
$$

and the convex function $\varphi: \mathbb{R}^{n} \rightarrow \mathbb{R}$ is defined by

$$
\varphi(x)=\left(\Phi \circ H_{1}^{T} M^{-\frac{1}{2}}\right)\left(x_{2}\right) .
$$

In this case, let us observe that the subdifferential of $\varphi$ is given by

$$
\partial \varphi(x)=\left(\begin{array}{c}
0_{m \times 1} \\
\partial\left(\Phi \circ H_{1}^{T} M^{-\frac{1}{2}}\right)\left(x_{2}\right)
\end{array}\right)=\left(\begin{array}{c}
0_{m \times 1} \\
M^{-\frac{1}{2}} H_{1} \partial \Phi\left(H_{1}^{T} M^{-\frac{1}{2}} x_{2}\right) .
\end{array}\right) .
$$


It is clear that $A$ is continuous and $A+\omega I_{n \times n}$ is monotone provided that $\omega \geq \sup _{\|x\|=1}\langle-A x, x\rangle$. As a direct consequence of Corollary 5.3, we obtain an existence result for second order periodic systems.

Theorem 6.1 If the function $\Phi$ is convex and Lipschitz continuous and $\operatorname{Re}(\sigma(A)) \subset] 0,+\infty[$, then there exists at least one $q \in C^{1}\left(0, T ; \mathbb{R}^{n}\right)$ such that $\frac{d^{2} q}{d t^{2}} \in L^{\infty}\left(0, T ; \mathbb{R}^{n}\right)$ satisfying $(6.3)$ (6.4).

Remark 6.1 We note that the conclusions of Theorem 6.1 hold under the key assumption that $\Phi$ is convex and Lipschitz continuous. Let us now give a counterexample when $\Phi$ is convex but not Lipschitz. Let us take $m=1, \Phi: \mathbb{R} \rightarrow \mathbb{R}, x \mapsto \Phi(x)=x^{2}, M=C=K=H_{1}=1$ and $F(t)=-t, \forall t \in[0,1]$. The differential inclusion (6.4) then reduces to

$$
\left\{\begin{array}{l}
\ddot{q}(t)+2 \dot{q}(t)+q(t)=t \\
q(0)=q(1) \\
\dot{q}(0)=\dot{q}(1)
\end{array}\right.
$$

We let the reader check that problem (6.10) has no solutions.

Example 6.1 Let us take $m=1, \Phi: \mathbb{R} \rightarrow \mathbb{R} ; x \mapsto \Phi(x)=|x|$. In this case, we have

$$
\partial \Phi(x)=\operatorname{Sign}(x)
$$

where

$$
\operatorname{Sign}(x):= \begin{cases}-1, & \text { if } x<0 \\ {[-1,+1],} & \text { if } x=0 \\ +1, & \text { if } x>0\end{cases}
$$

We consider the following problem

$$
\left\{\begin{array}{l}
m \ddot{q}(t)+c \dot{q}(t)+k q(t)-F(t) \in-\operatorname{Sign}(\dot{q}(t)), \quad t \in[0, T] \\
q(0)=q(T), \\
\dot{q}(0)=\dot{q}(T)
\end{array}\right.
$$

with $F \in C^{0}\left(\left[0,+\infty[; \mathbb{R})\right.\right.$ such that $\frac{d F}{d t} \in L_{\text {loc }}^{1}([0,+\infty[; \mathbb{R})$. The matrix $A$ in $(6.5)$ is given by

$$
A=\left(\begin{array}{cc}
0 & -1 \\
\frac{k}{m} & \frac{c}{m}
\end{array}\right) \text {. }
$$

We suppose that $m, c, k>0$ and set $\Delta=\frac{c^{2}}{m^{2}}-4 \frac{k}{m}$. Then we have

$$
\sigma(A)= \begin{cases}\left\{\frac{c}{2 m}-\frac{\sqrt{\Delta}}{2}, \frac{c}{2 m}+\frac{\sqrt{\Delta}}{2}\right\}, & \text { if } c \geq 2 k \sqrt{m}, \\ \left\{\frac{c}{2 m}-i \frac{\sqrt{-\Delta}}{2}, \frac{c}{2 m}+i \frac{\sqrt{-\Delta}}{2}\right\}, & \text { if } c<2 k \sqrt{m} .\end{cases}
$$

We note that in both cases $\operatorname{Re}(\sigma(A)) \subset] 0,+\infty$ [ and hence by Theorem 6.1 , problem (6.11) has at least a solution. 


\section{References}

[1] Adly, S., Attractivity theory for second order dynamical systems with application to dry friction, Journal of Math. Anal. and Appl., 322, 2006, 1055-1070.

[2] Adly, S. and D. Goeleven, D., A stability theory for second order non-smooth dynamical systems with application to friction problems, J. Math. Pures Appl., 83, 2004, 17-51.

[3] Amann, H., A note on degree theory for gradient mappings, Proc. Amer. Math. Soc., 85, 1982, 591-595.

[4] Brézis, H., Problèmes unilatéraux, J. Math. Pures Appl., 51, 1972, 1-168.

[5] Brézis, H., Opérateurs Maximaux Monotones et Semi-groupes de Contractions dans les Espaces de Hilbert, North Holland, Amsterdam, 1973.

[6] de Blasi, F., Górniewicz, L. and Pianigiani, G., Topological degree and periodic solutions of differential inclusions, Nonlinear Anal. Ser. A: Theory Methods, 37(2), 1999, 217-243.

[7] Goeleven, D. and Brogliato, B., Necessary conditions of asymptotic stability for unilateral dynamical systems, Nonlinear Analysis, 61, 2005, 961-1004.

[8] Goeleven, D., Motreanu, D. and Motreanu, V., On the stability of stationary solutions of first order evolution variational inequalities, Advances in Nonlinear Variational Inequalities, 6, 2003, 1-30.

[9] Goeleven, D. and Brogliato, B., Stability and unstability matrices for linear evolution variational inequalities, IEEE Transaction on Automatic Control, 49, 2004, 1-14.

[10] Górniewicz, L., Topological approach to differential inclusions, Topological Methods in Differential Equations and Inclusions, A. Granas and M. Frigon (eds.), NATO ASI Series, Mathematical and Physical Sciences, Vol. 472, Kluwer Academic Publishers, 1994.

[11] Hiriart-Urruty, J. B. and Lemaréchal, C., Fundamentals of Convex Analysis, Springer, Berlin, 1993.

[12] Kinderlehrer, D. and Stampacchia, G., An Introduction to Variational Inequalities and Their Applications, Academic Press, New York, 1980.

[13] Krasonel'skii, M. A., The Operator of Translation along the Trajectories of Differential Equations, Nauka, Moscow, 1966 (in Russian); English translation: American Math. Soc., Translations of Math. Monographs, Vol. 19, Providence, 1968.

[14] Krasonel'skii, M. A. and Zabreiko, P. P., Geometrical Methods of Nonlinear Analysis, Nauka, Moscow, 1975 (in Russian); English translation: Springer, Berlin, 1984.

[15] Lions, J. L., Quelques Méthodes de Résolution des Problèmes aux Limites Non-linéaires, Dunod, Paris, 1969.

[16] Lloyd, N. G., Degree Theory, Cambridge University Press, Cambridge, 1978.

[17] Mawhin, J., Continuation theorems and periodic solutions of ordinary differential equations, Topological Methods in Differential Equations and Inclusions, A. Granas and M. Frigon (eds.), NATO ASI Series, Mathematical and Physical Sciences, Vol. 472, Kluwer Academic Publishers, 1994.

[18] Showalter, R. E., Monotone Operators in Banach Spaces and Nonlinear Partial Differential Equations, American Mathematical Society, Providence, RI, USA, 1997.

[19] Zeidler, E., Nonlinear Functional Analysis and Its Applications, I, Fixed-Point Theorems, Springer-Verlag, New York, 1986. 